\title{
A family of smooth and interpolatory basis functions for parametric curve and surface representation ${ }^{\text {th }}$
}

\author{
D. Schmitter*, R. Delgado-Gonzalo, M. Unser \\ Biomedical Imaging Group, EPFL, Switzerland
}

\section{A R T I C L E I N F O}

\section{Article history:}

Available online 22 July 2015

\section{Keywords:}

B-splines

Exponential B-splines

Compact support

Interpolation

Parametric curves

Parametric surfaces

\begin{abstract}
A B S T R A C T
Interpolatory basis functions are helpful to specify parametric curves or surfaces that can be modified by simple user-interaction. Their main advantage is a characterization of the object by a set of control points that lie on the shape itself (i.e., curve or surface). In this paper, we characterize a new family of compactly supported piecewise-exponential basis functions that are smooth and satisfy the interpolation property. They can be seen as a generalization and extension of the Keys interpolation kernel using cardinal exponential B-splines. The proposed interpolators can be designed to reproduce trigonometric, hyperbolic, and polynomial functions or combinations of them. We illustrate the construction and give concrete examples on how to use such functions to construct parametric curves and surfaces.
\end{abstract}

(c) 2015 Elsevier Inc. All rights reserved.

\section{Introduction}

The representation of shapes using parametric curves and surfaces is widely used in domains that make use of computer graphics [1,2] such as industrial design [3-5], the animation industry [6], as well as for the analysis of biomedical images [7-9]. In that context, it is often important to be able to interactively change the shape of the curve or surface. The spline-based representation of parametric shapes has proven to be a convenient choice to include user interactivity in shape modeling due to the underlying control-point-based nature of spline functions. If the basis functions are compactly supported, the change of position of a control point modifies the shape only locally. This allows for a local control by the user. Commonly used basis functions such as NURBS or B-splines have this locality property but are in general not interpolatory (except for example zeroth and first degree B-splines, which are not smooth) [10]. This has the disadvantage that the control points do not directly lie on the contour or surface of the shape. Especially in 3D applications, this can be inconvenient because it is no longer intuitive to interactively modify complex shapes. More recently a method to construct piecewise polynomial interpolators has been presented in [11,12].

In this paper, we propose a new family of piecewise exponential basis functions that are interpolatory and are at least in $\mathcal{C}^{1}$. They are compactly supported and their order can be chosen to be arbitrarily high. We show that they are able to reproduce exponential polynomials which include the pure polynomials as a subset. This convenient property is particularly relevant for the exact rendering of conic sections such as circles, ellipses, or parabolas, as well as other trigonometric and hyperbolic curves and surfaces [13]. In its absence, one must resort to subdivision to tackle this aspect [14-18]. However, existing comparable subdivision schemes usually rely on basis functions that are defined as a limit process and do not have a closed-form expression [19].

\footnotetext{
This work was funded by the Swiss National Science Foundation under grant 200020-144355.

* Corresponding author. Tel.: +4121 6935135.

E-mail address: daniel.schmitter@epfl.ch (D. Schmitter).
} 
Our proposed family generalizes the piecewise-polynomial Keys interpolator [20-22] to higher degrees and can be seen as its extension using exponential B-splines $[23,24]$.

The paper is organized as follows. In Section 2 we give a brief review on exponential B-splines and their relation with differential operators. This is needed to understand the reproduction properties of our proposed interpolators since they are based on exponential B-splines. In Section 3 we present the proposed family of interpolators. We present the relevant properties and prove that they reproduce exponential polynomials. We also provide a generic algorithm to construct specific interpolators that belong to the proposed family. In Section 4 we give specific examples of interpolators and we explicitly show how parametric curves and surfaces with desirable reproduction properties are constructed.

\section{Exponential B-splines}

In this section, we briefly review the link between exponential B-splines and differential operators which is crucial to understand the reproduction properties of the proposed spline family. (For a more in-depth characterization of exponential B-splines, we refer the reader to $[23,24]$.) These reproduction properties are needed for the exact representation of elementary shapes (see Section 4.3 for examples) and are automatically enforced by our construction.

\subsection{Notations}

We describe the list of roots $\alpha_{1}, \ldots, \alpha_{N}$ using the vector notation $\alpha=\left(\alpha_{1}, \alpha_{2}, \ldots, \alpha_{N}\right)$. To assert the inclusion of a list of roots $\alpha_{1}$ into another list $\alpha_{2}$, we use the set notation $\alpha_{1} \subset \alpha_{2}$. If $\alpha_{1}$ must be excluded from $\alpha_{2}$, we write $\alpha_{2} \backslash \boldsymbol{\alpha}_{1}$. Similarly, we denote the union of the two lists of roots $\alpha_{1}$ and $\alpha_{2}$ by $\alpha_{1} \cup \alpha_{2}$. Likewise, we write $\alpha_{n} \in \boldsymbol{\alpha}$ to signify that one of the components of $\alpha$ is $\alpha_{n}$. Furthermore, the nth-order derivative operator is denoted by $D^{n}=\frac{\mathrm{d}^{n}}{\mathrm{~d} t^{n}}$ with $D^{0}=I$ (identity operator).

\subsection{Operator properties and reproduction of null-space components}

Consider the generic differential operator $L$ of order $N$

$$
L=D^{N}+a_{N-1} D^{N-1}+\cdots+a_{0} I .
$$

Its characteristic polynomial with variable $s \in \mathbb{C}$ is given by

$$
L(s)=s^{N}+a_{N-1} s^{N-1}+\cdots+a_{0}=\prod_{n=1}^{N}\left(s-\alpha_{n}\right) .
$$

By evaluating $L(s)$ at $s=\mathrm{j} \omega$, where $\mathrm{j}^{2}=-1$, we obtain that the frequency response of the differential operator is $\hat{L}(\mathrm{j} \omega)=$ $\prod_{n=1}^{N}\left(\mathrm{j} \omega-\alpha_{n}\right)$. This allows us to factorize the operator $L$ as

$$
L_{\alpha}:=L=\left(D-\alpha_{1} I\right)\left(D-\alpha_{2} I\right) \cdots\left(D-\alpha_{N} I\right) .
$$
by

It follows that the nullspace, which contains all the solutions of the homogenous differential equation $L_{\alpha}\left\{f_{0}\right\}(t)=0$, is given

$$
N_{L_{\alpha}}=\operatorname{span}\left\{t^{n-1} \mathrm{e}^{\alpha_{(m)} t}\right\}_{m=1, \ldots, N_{d} ; n=1, \ldots, n_{(m)}},
$$

where the $N_{d}$ distinct roots of the characteristic polynomial are denoted by $\alpha_{(1)}, \ldots, \alpha_{\left(N_{d}\right)}$ with the multiplicity of $\alpha_{(m)}$ being $n_{(m)}$ and $\sum_{m=1}^{N_{d}} n_{(m)}=N$. There exists a unique causal Green's function $\rho_{\boldsymbol{\alpha}}\left(\rho_{\boldsymbol{\alpha}}(t)=0\right.$ for $\left.t<0\right)$ associated to the operator $L_{\boldsymbol{\alpha}}$ that satisfies $L_{\alpha}\left\{\rho_{\alpha}\right\}(t)=\delta(t)$, where $\delta$ is the Dirac distribution.

Its explicit form is

$$
\rho_{\boldsymbol{\alpha}}(t)=\sum_{m=1}^{N_{d}} \sum_{n=1}^{n_{(m)}} c_{m, n} \frac{t_{+}^{n-1}}{(n-1) !} \mathrm{e}^{\alpha_{(m)} t},
$$

with suitable constants $c_{m} n$. We see that (5) is a causal exponential polynomial. The discrete counterpart of $L_{\alpha}$ is denoted by $\Delta_{\alpha}$. It is specified by its symbol $\hat{\Delta}_{\alpha}(z)=\prod_{n=1}^{N}\left(1-\mathrm{e}^{\alpha_{n}} z^{-1}\right)$. An exponential B-spline is then defined as $\beta_{\alpha}(t)=\Delta_{\alpha}\left\{\rho_{\alpha}\right\}(t)$, which is equivalent to the Fourier-domain definition

$$
\hat{\beta}_{\alpha}(\omega)=\frac{\hat{\Delta}_{\alpha}\left(\mathrm{e}^{\mathrm{j} \omega}\right)}{\hat{L}_{\alpha}(\mathrm{j} \omega)}=\prod_{k=1}^{n} \frac{1-\mathrm{e}^{\alpha_{k}-\mathrm{j} \omega}}{\mathrm{j} \omega-\alpha_{k}} .
$$

Since $\Delta_{\alpha}$ is defined on the integer grid, the exponential B-splines reproduce the causal Green's function (5) associated to $L_{\alpha}$

$$
\rho_{\alpha}(t)=\Delta_{\alpha}^{-1}\left\{\beta_{\alpha}\right\}(t)=\sum_{k=0}^{+\infty} p_{\alpha}[k] \beta_{\alpha}(t-k),
$$

where $p_{\alpha}$ is a unique causal sequence as has been shown in [23]. Extrapolating the Green's function (5) for $t<0$ is equivalent to extrapolating the sum in (7) for negative $k$, which results in the reproduction of an exponential polynomial. More generally, it can be shown that $\beta_{\alpha}$ is able to reproduce any component $P_{0}(t) \in N_{L}$ that is in the null space of $L=L_{\alpha}$. 


\section{Construction of interpolatory basis functions}

\subsection{Desirable properties of the basis functions}

We want to construct an interpolator based on a suitable linear combination of exponential B-splines of different orders. The following characteristics should be met:

\section{- Smoothness}

We want the interpolation functions to be at least continuously differentiable and, hence, the minimum order of the B-spline involved is 3 (i.e., degree 2).

- Support

The interpolator should be compactly supported and the support of the function should not be larger than the support of the B-spline of highest order $N$ involved. Therefore, the support of the resulting function is an integer and is equal to $N$.

- Symmetry We want the interpolator to be symmetric. This can be achieved if the non-zero poles of the exponential spline are grouped in pairs of opposite sign [23]. Furthermore, except for the highest-order B-spline involved, the B-splines in the sum have to come in pairs and be shifted accordingly.

- Interpolation condition

The constructed function has to satisfy the interpolation condition

$$
\left.\varphi(t)\right|_{t=k}=\delta[k], \quad k \in \mathbb{Z},
$$

where $\delta[k]$ represents the Kronecker delta.

- Reproduction of exponential polynomials

We are interested in representing shapes that do not only rely on polynomial but also on trigonometric and hyperbolic coordinate functions. Thus, the interpolators must reproduce exponential polynomials.

\subsection{Characterization of the family of interpolators}

Taking all of the above considerations into account we characterize an Nth order smooth and piecewise exponential interpolator as

$$
\varphi(t):=\lambda_{N} \beta_{\boldsymbol{\alpha}_{N}}\left(t+\frac{N}{2}\right)+\sum_{n=n_{0}}^{N-1} \lambda_{n}\left(\beta_{\boldsymbol{\alpha}_{n}}\left(t+\frac{N}{2}\right)+\beta_{\boldsymbol{\alpha}_{n}}\left(t-\frac{N}{2}+n\right)\right),
$$

where $\boldsymbol{\alpha}_{n_{0}}$ has at least $n_{0}=3$ poles (smoothness constraint) and $N \geq 2\left(n_{0}-1\right)$ is an integer that defines the highest-order exponential B-spline involved. Furthermore, in order for $\varphi$ to reproduce exponential polynomials, we enforce $\boldsymbol{\alpha}_{n} \subset \boldsymbol{\alpha}_{N}$ for $n \in\left[n_{0}, N-1\right]$ (see Section 3.4.1). Here, the notation $\boldsymbol{\alpha}_{n}$ implies that the list of poles $\boldsymbol{\alpha}_{n}$ contains $n$ elements. Hence, using the fact that the support of an exponential B-spline is equal to the number of poles that specifies it, we see that $\varphi$ is of support equal to $N$.

The weights $\lambda_{n}$ are computed by making use of the symmetry of the interpolator, i.e., $\varphi(t)=\varphi(-t)$ and by imposing the interpolation constraints (8), which we achieve by solving the system of equations

$$
\left\{\begin{array}{l}
1=\varphi(0) \\
0=\varphi(1) \\
\vdots \\
0=\varphi(\lfloor N / 2\rfloor) .
\end{array}\right.
$$

From (10) we see that, $N / 2$ (even case) respectively $N / 2+1$ (odd case) interpolation constraints have to be met to construct the function. This follows from the fact that $\varphi$ can only be non-zero within the interval $[-N / 2, N / 2]$. If $N$ is even, $N / 2$ is integer and since the interpolator is smooth $\varphi(N / 2)=0$ and hence, does not explicitly need to be imposed in (10).

\subsection{Construction of basis functions}

The proposed interpolatory basis functions are constructed as follows:

1. Define an exponential spline type with desirable reproduction properties; that is, select the list $\boldsymbol{\alpha}_{n_{0}}$ that contains the featured poles and whose total number of elements is $n_{0} \geq 3$.

2. Given $\boldsymbol{\alpha}_{n_{0}}, N$ must be no smaller than $N_{\min }=2\left(n_{0}-1\right)$. This restriction is directly related to the interpolation constraints.

3. The Nth-order interpolator is given by (9), where $\boldsymbol{\alpha}_{n}=\boldsymbol{\alpha}_{n_{0}} \cup \mathbf{0}_{n-n_{0}}$ with $n>n_{0}$ and $\boldsymbol{0}_{K}$ is the $K$ element vector filled with zeros.

4. The weights $\lambda_{n}$ can be computed by solving (10). 
The system (10) is overdetermined when $N>N_{\min }$. In that case, the weights $\lambda_{n}$ for $\left(N-N_{\min }\right)$ terms in (9) can be chosen arbitrarily (see Section 4.3 for examples). Conversely, we see that, in order to satisfy the interpolation constraints for a given order $N$, the smallest possible number of poles is $n_{0}=\lfloor N / 2\rfloor+1$. Otherwise, when the system of equations is overdetermined, one can always construct $\boldsymbol{\alpha}_{n_{0}^{\prime}}=\boldsymbol{\alpha}_{n_{0}} \cup \mathbf{0}_{(\lfloor N / 2\rfloor+1)-n_{0}}$ with $n_{0}^{\prime}$ poles. The resulting exponential B-spline $\beta_{\boldsymbol{\alpha}_{n_{0}^{\prime}}}$ preserves the reproduction properties of $\beta_{\boldsymbol{\alpha}_{n_{0}}}$ but has increased order of approximation and regularity [23] (see Section 3.4). Based on our experiments we conjecture that the system of equations (10) always has a solution.

\subsection{Reproduction properties and regularity}

\subsubsection{Reproduction of exponential polynomials}

Proposition 1. The interpolator defined by (9) reproduces exponential polynomials up to degree $q$ and exponent $\alpha$ if and only if $\boldsymbol{\alpha}_{n_{0}}$ contains $q+1$ copies of $\alpha$.

Proof. We first show that exponential polynomials can be reproduced with exponential B-splines and then conclude that $\varphi$ preserves these reproduction properties.

An exponential polynomial of exponent $\alpha$ and degree $q$ can always be written as a linear combination of exponential monomials $Q_{\alpha}^{n}(t)=\mathrm{e}^{\alpha t} t^{n}$ for $n=0, \ldots, q$. The exponential polynomial is expressed as

$$
P_{\alpha}^{q}(t)=\sum_{k=0}^{q} a_{k} \mathrm{e}^{\alpha t} t^{k} .
$$

For $n \geq n_{0}$ in (9) and $n \in\left[n_{0}, N\right]$, every exponential B-spline $\beta_{\boldsymbol{\alpha}_{n}}$ is defined through a list $\boldsymbol{\alpha}_{n}$ that contains $\boldsymbol{\alpha}_{n_{0}}$. Furthermore, from [23] we know that, for $\alpha \in \boldsymbol{\alpha}_{n_{0}}$ of multiplicity $q+1$, there exist sequences $p_{n}$ such that

$$
\mathrm{e}^{\alpha t} t^{n}=\sum_{k \in \mathbb{Z}} p_{n}[k] \beta_{\boldsymbol{\alpha}_{n_{0}}}(t-k)
$$

for $n=0, \ldots, q$, which is equivalent to saying that $\beta_{\boldsymbol{\alpha}_{n_{0}}}$ reproduces exponential monomials up to degree $q$ and exponent $\alpha$. The shifted exponential B-splines in (9) also have the same reproduction property. By combining (11) and (12) and considering an arbitrary shift $m$, we see that

$$
\begin{aligned}
P_{\alpha}^{n}(t-m) & =\sum_{k=0}^{n} a_{k} Q_{\alpha}^{k}(t-m) \\
& =\sum_{k=0}^{n} a_{k} \sum_{l \in \mathbb{Z}} p_{k}[l] \beta_{\alpha_{n_{0}}}(t-m-l) \\
& =\sum_{k=0}^{n} a_{k} \mathrm{e}^{\alpha(t-m)}(t-m)^{k} \\
& =\mathrm{e}^{\alpha t} \sum_{k=0}^{n} a_{k} \mathrm{e}^{-\alpha m} \sum_{l=0}^{k}\left(\begin{array}{l}
k \\
l
\end{array}\right) t^{l}(-1)^{k-l} m^{k-l},
\end{aligned}
$$

which is a linear combination of polynomials in $t$ of degree up to $n$ that are multiplied by $\mathrm{e}^{\alpha t}$. Thus, we can collect all the factors multiplying $t^{k}$ and rewrite them as $b_{k}$ to express (13) as

$$
P_{\alpha}^{n}(t-m)=\mathrm{e}^{\alpha t} \sum_{k=0}^{n} b_{k} t^{k}:=P_{\alpha, m}^{n}(t)
$$

for $n=0, \ldots, q$, which is also an exponential polynomial of exponent $\alpha$ and degree $n$.

In the next step, we first show that exponential polynomials can be reproduced if $\varphi$ is composed of exponential B-splines of identical degree and containing the same poles. Then we conclude that, because the reproduction of exponential polynomials is preserved through convolution, $\varphi$ also reproduces these exponential polynomials.

By (12) and (14) and using exponential B-splines of the same degree, we write

$$
\begin{aligned}
\sum_{k=0}^{p} & a_{k} \sum_{l \in \mathbb{Z}} p_{k}[l]\left(\lambda_{N} \beta_{\alpha_{n_{0}}}\left(t+\frac{N}{2}-l\right)+\sum_{n=\lfloor N / 2\rfloor+1}^{N-1} \lambda_{n}\left(\beta_{\alpha_{n_{0}}}\left(t+\frac{N}{2}-l\right)+\beta_{\alpha_{n_{0}}}\left(t-\frac{N}{2}+n-l\right)\right)\right) \\
= & P_{\alpha,-\frac{N}{2}}^{p}(t)\left(\lambda_{N}+\sum_{n=\lfloor N / 2\rfloor+1}^{N-1} \lambda_{n}\right)+\sum_{n=\lfloor N / 2\rfloor+1}^{N-1} \lambda_{n} P_{\alpha, \frac{N}{2}-n}^{p}(t)
\end{aligned}
$$

for $p=0, \ldots, q$, which is also an exponential polynomial with the same degree and exponent as its constituents.

The next step of the proof relies on a proposition originally stated by Unser and Blu in [23], which we recall here for the sake of completeness. 
Proposition. (Unser and Blu [23]) Let $\psi_{\alpha}$ be a function that reproduces the exponential polynomials in $\operatorname{span}\left\{\mathrm{e}^{\alpha t}, \ldots, t^{p} \mathrm{e}^{\alpha t}\right\}$. Then, for any compactly supported function $\psi$ such that $\int_{\mathbb{R}} \psi(t) \mathrm{e}^{-\alpha t} \mathrm{~d} t \neq 0$, the composite function $\psi * \psi_{\alpha}$ also reproduces these exponential polynomials (where $*$ denotes the convolution product).

Using this proposition, we deduce that, for $n \in\left[n_{0}, N\right]$, the convolution product

$$
\beta_{\boldsymbol{\alpha}_{n} \backslash \boldsymbol{\alpha}_{n_{0}}} * \beta_{\boldsymbol{\alpha}_{n_{0}}}
$$

preserves the exponential reproduction properties of $\beta_{\boldsymbol{\alpha}_{n_{0}}}$. Note that in (16), the term $\beta_{\boldsymbol{\alpha}_{n_{0}} \backslash \boldsymbol{\alpha}_{n_{0}}}=1$.

From the definition of the interpolatory basis function (9) and by combining (15) and (16), we obtain

$$
\begin{aligned}
\sum_{k=0}^{p} a_{k} \sum_{l \in \mathbb{Z}} \tilde{p}_{k}[l] \varphi(t-l)= & \sum_{k=0}^{p} a_{k} \sum_{l \in \mathbb{Z}} \tilde{p}_{k}[l]\left(\lambda_{N} \beta_{\boldsymbol{\alpha}_{N}}\left(t+\frac{N}{2}-l\right)+\sum_{n=\lfloor N / 2\rfloor+1}^{N-1} \lambda_{n}\left(\beta_{\boldsymbol{\alpha}_{n}}\left(t+\frac{N}{2}-l\right)+\beta_{\boldsymbol{\alpha}_{n}}\left(t-\frac{N}{2}+n-l\right)\right)\right) \\
= & \sum_{k=0}^{p} a_{k} \sum_{l \in \mathbb{Z}} \tilde{p}_{k}[l]\left(\lambda_{N}\left(\beta_{\boldsymbol{\alpha}_{N} \backslash \boldsymbol{\alpha}_{n_{0}}} * \beta_{\boldsymbol{\alpha}_{n_{0}}}\right)\left(t+\frac{N}{2}-l\right)\right. \\
& \left.+\sum_{n=\lfloor N / 2\rfloor+1}^{N-1} \lambda_{n}\left(\beta_{\boldsymbol{\alpha}_{n} \backslash \boldsymbol{\alpha}_{n_{0}}} *\left(\beta_{\boldsymbol{\alpha}_{n_{0}}}+\beta_{\boldsymbol{\alpha}_{n_{0}}}(\cdot-N)\right)\right)\left(t+\frac{N}{2}+n-l\right)\right)
\end{aligned}
$$

where $\tilde{p}_{k}$ is a suitable sequence of coefficients. Therefore, from (17) we see that $\varphi$ also reproduces the exponential polynomials given by (15) up to degree $q$ and exponent $\alpha$, where $\alpha \in \boldsymbol{\alpha}_{n_{0}}$ is of multiplicity $q+1$.

\subsubsection{Regularity}

The regularity of the proposed interpolator depends on the exponential B-spline of lowest order that is involved in the construction of $\varphi$. Hence, $\varphi$ belongs to $\mathcal{C}^{n_{0}-2}$.

\subsubsection{Order of approximation}

If the poles of the constructed interpolators are of the form $\alpha=\frac{\pi x}{M}, x \in \mathbb{C}$, and if $M$ is related to the number of control points, then the definition of the interpolator (9) implies that, as $M \rightarrow \infty, \varphi$ converges to piecewise-polynomial interpolators that have an $\left(n_{0}\right)$ th order of approximation (by the Strang-Fix equivalence $\left.[25,26]\right)$. Such interpolators are of special interest for the construction of particular shapes (see Section 4.3).

In the following, we give concrete examples of interpolatory basis functions that are derived from (9).

\section{Examples of interpolators and applications}

\subsection{Polynomial bases}

If the pole vector entirely consists of zeroes, the basis function is a sum of polynomial B-splines and hence is piecewise polynomial. For example, the 4th-order basis corresponds to the Keys interpolation kernel [20]. These basis functions are all symmetric. Some examples are shown in Fig. 1.

\subsection{Trigonometric and hyperbolic bases}

Trigonometric and hyperbolic functions take special relevance within computational geometry. Exponential splines that are able to reproduce (hyberbolic) sines and cosines can be used to construct the desired interpolatory basis functions. Because the exponents involved in the representation of (hyperbolic) sinusoidal functions come in pairs of opposite sign, the resulting basis functions are symmetric as a consequence of the symmetry of their elementary constituents. Some hyperbolic and trigonometric interpolators are shown in Fig. 2.

\subsection{Larger support interpolators}

For the sake of completeness, we also provide an example of how to construct interpolators by choosing $n_{0}$ and $N$ such that the corresponding system of equations is overdetermined (see Section 3.3). Such situations arise if either for a given $\boldsymbol{\alpha}_{n_{0}}$ a corresponding $N>N_{\min }=2\left(n_{0}-1\right)$ is chosen or if for a given $N$ a corresponding $n_{0}<\lfloor N / 2\rfloor+1$ is chosen. In both cases, the weights $\lambda_{n}$ for $\left(N-N_{\min }\right)$ terms in (9) can be chosen arbitrarily. Our experiments show that such interpolators that are constructed by solving an overdetermined system of equations tend to oscillate more than their "smallest" support counterparts. Examples are shown in Fig. 3 (middle and right), where we constructed 6th- and 7th-order interpolators.

\subsubsection{Applications}

In this section, we show how idealized parametric curves and surfaces (such as ellipses and ellipsoids) can be reproduced using the proposed interpolators. Such shapes can be constructed independently of the number of control points, which makes them particularly useful for deformable models where, when starting from an initial configuration, it is desirable to approximate shapes with arbitrary precision $[8,9,13,27]$. We construct symmetric interpolators that have the smallest support given $\boldsymbol{\alpha}_{n_{0}}$ as described in Section 3.3. 


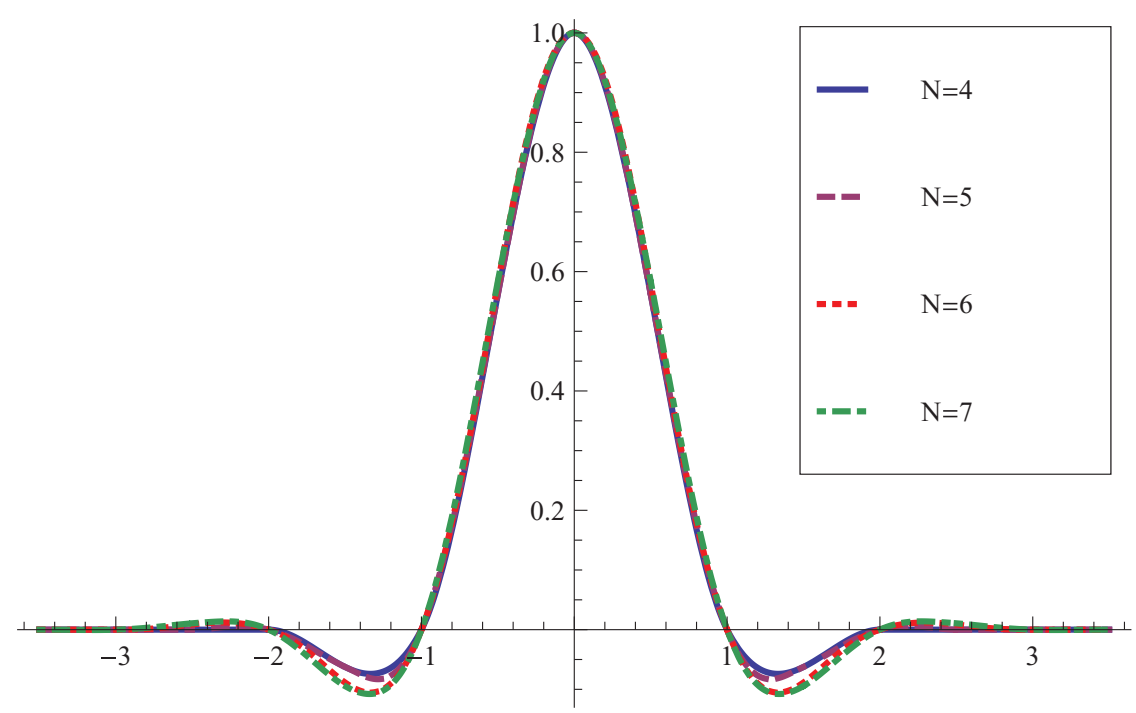

Fig. 1. Examples of polynomial-reproducing interpolators. Their poles are all equal to zero and their respective order corresponds to $N=4, \ldots, 7$, and is equal to their support. The basis function that corresponds to $N=4$ represents the Keys interpolator [20].
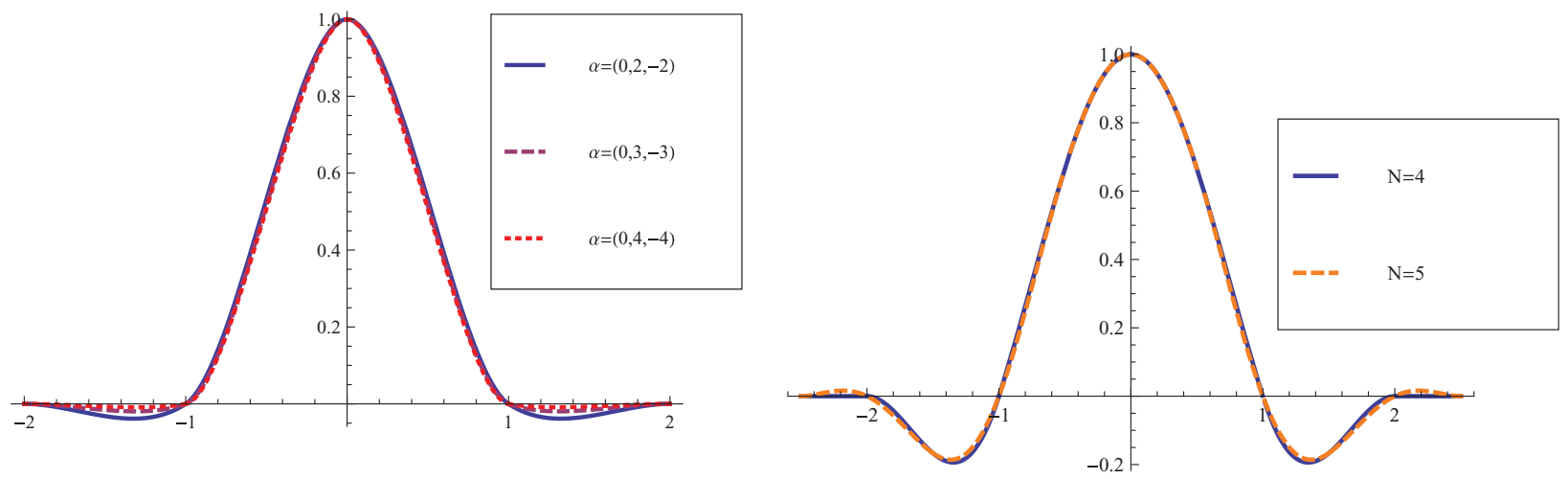

Fig. 2. Hyperbolic (left) and trigonometric (right) interpolators. Left: 4th-order interpolating functions are shown that were constructed with different lists of poles indicated by $\boldsymbol{\alpha}$. Right: Trigonometric interpolators. The two interpolators correspond to 4 th and 5 th order with $\boldsymbol{\alpha}_{n_{0}}=\left(0, \frac{2 j \pi}{3},-\frac{2 \mathrm{j} \pi}{3}\right)$.
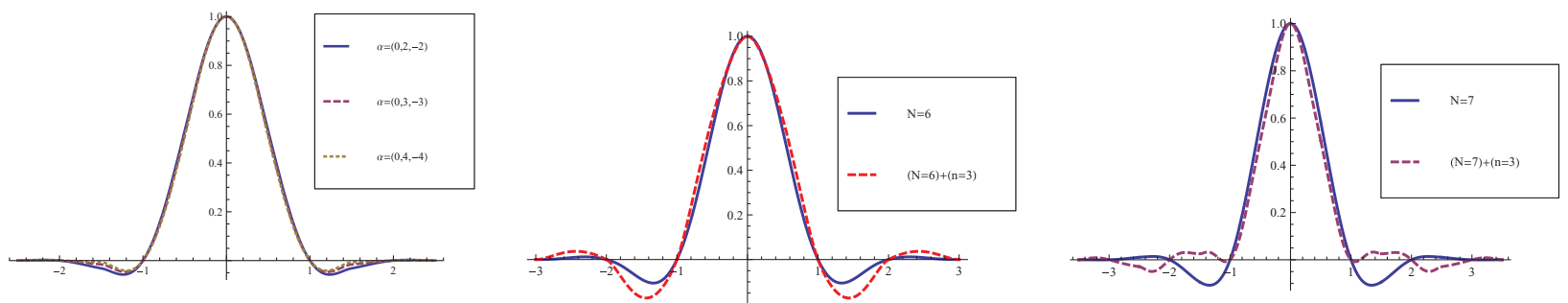

Fig. 3. Larger support interpolators. Left: Hyperbolic interpolators; 5th-order interpolators are shown that were constructed with different lists of poles $\boldsymbol{\alpha}$. Regarding the number of elements in $\boldsymbol{\alpha}$ which is equal to 3, their "smallest" support counterparts (which are shown in Fig. 2) are constructed with $N_{\text {min }}=$ 2(3-1) $=4$, whereas here $N=5$ was chosen. Middle and right: Effect of including lower-order B-splines in the construction of the interpolating function. If the order of the interpolator is $N$, then the lowest-order B-spline involved in its construction must be at least $n=\lfloor N / 2\rfloor+1$ (blue curves). If splines of lower order than $n=\lfloor N / 2\rfloor+1$ are used in the construction, the interpolating function shows an oscillatory behavior (red and magenta curves respectively). Here we have used $N=6$ (middle) and $N=7$ (right) respectively and hence, the required minimum-order B-spline involved corresponds to $n=n_{0}^{\prime}=\lfloor 6 / 2\rfloor+1=$ $\lfloor 7 / 2\rfloor+1=4$ and the corresponding list of poles is $\boldsymbol{\alpha}=(0,0,0,0)=\boldsymbol{\alpha}_{n_{0}^{\prime}}=\boldsymbol{\alpha}_{n_{0}} \cup \mathbf{0}_{(\lfloor N / 2\rfloor+1)-n_{0}}=\boldsymbol{\alpha}_{n_{0}} \cup \mathbf{0}_{4-3}=(0,0,0) \cup(0)$. In the construction of the red and magenta interpolators we have additionally used a B-spline of order $n=3$ which corresponds to $\boldsymbol{\alpha}=(0,0,0)=\boldsymbol{\alpha}_{n_{0}}$. (For interpretation of the references to color in this figure, the reader is referred to the web version of this article.) 


\subsection{Reproduction of parametric curves}

\subsubsection{Reproduction of ellipses}

Here we explicitly show how ellipses are reproduced within our framework. We consider the lowest order, which is $N=4$. The condition for being able to reconstruct an ellipse with $M$ control points is $\boldsymbol{\alpha}_{n_{0}}=\boldsymbol{\alpha}_{3}:=\left(0, \frac{\mathrm{j} 2 \pi}{M},-\frac{\mathrm{j} 2 \pi}{M}\right)$. Therefore, by applying (9), the interpolator is

$$
\varphi(t)=\lambda_{4} \beta_{\boldsymbol{\alpha}_{4}}(t+2)+\lambda_{3}\left(\beta_{\boldsymbol{\alpha}_{3}}(t+2)+\beta_{\boldsymbol{\alpha}_{3}}(t+1)\right)
$$

with $\boldsymbol{\alpha}_{4}=\left(0,0, \frac{\mathrm{j} 2 \pi}{M},-\frac{\mathrm{j} 2 \pi}{M}\right)$ and the $\lambda_{n}$ which are found by solving (10). Specifically, we end up with the system of equations

$$
\left\{\begin{array}{l}
0=\lambda_{4} \beta_{\alpha_{4}}(1)+\lambda_{3}\left(\beta_{\alpha_{3}}(1)+\beta_{\alpha_{3}}(0)\right)=\lambda_{4} \beta_{\alpha_{4}}(1)+\lambda_{3} \beta_{\alpha_{3}}(1) \\
1=\lambda_{4} \beta_{\alpha_{4}}(2)+\lambda_{3}\left(\beta_{\alpha_{3}}(2)+\beta_{\alpha_{3}}(1)\right)=\lambda_{4} \beta_{\alpha_{4}}(2)+2 \lambda_{3} \beta_{\alpha_{3}}(2)
\end{array}\right.
$$

whose solution is

$$
\lambda_{3}(M)=\frac{\pi^{2} \csc \left(\frac{\pi}{M}\right) \csc \left(\frac{2 \pi}{M}\right)\left(M-2 \pi \csc \left(\frac{2 \pi}{M}\right)\right)}{M^{2}\left(M \sec \left(\frac{\pi}{M}\right)-\pi \csc \left(\frac{\pi}{M}\right)\right)}
$$

and

$$
\lambda_{4}(M)=\frac{\pi^{3} \sec ^{2}\left(\frac{\pi}{M}\right)}{M^{2}\left(M \tan \left(\frac{\pi}{M}\right)-\pi\right)} .
$$

To reproduce $\cos \left(\frac{2 \pi}{M} \cdot\right)$, we take advantage of the interpolation property which yields

$$
\cos \left(\frac{2 \pi}{M} t\right)=\sum_{k \in \mathbb{Z}} \frac{\mathrm{e}^{\mathrm{j} \frac{2 \pi}{M} k}+\mathrm{e}^{-\mathrm{j} \frac{2 \pi}{M} k}}{2} \varphi(t-k),
$$

where the coefficients are the integer samples of the curve. Normalizing the period of the cosine and using the $M$-periodized basis functions

$$
\varphi_{M}(M t-k)=\sum_{n=-\infty}^{+\infty} \varphi(M(t-n)-k),
$$

we express the cosine as

$$
\cos (2 \pi t)=\sum_{k=0}^{M-1} \cos \left[\frac{2 \pi k}{M}\right] \varphi_{M}(M t-k) .
$$

In a similar way we obtain

$$
\sin (2 \pi t)=\sum_{k=0}^{M-1} \sin \left[\frac{2 \pi k}{M}\right] \varphi_{M}(M t-k) .
$$

Plots of the trigonometric functions are shown in Fig. 4 as well as the circle obtained through the parametric equation $\boldsymbol{r}(t)=$ $(\cos (2 \pi t), \sin (2 \pi t))$.

Due to the choice of $\boldsymbol{\alpha}_{n_{0}}$, we see that, as we increase the number $M$ of control points, $\varphi$ converges to the 4th-order polynomial basis, which corresponds to the Keys interpolator.

\subsection{Reproduction of parametric surfaces}

\subsubsection{Sphere}

We can also reproduce spheres or ellipsoids by using the basis function defined in (18). Similar to [9], a possible parameterization of the sphere is given by

$$
\begin{aligned}
\sigma(u, v) & =\left(\begin{array}{l}
x(u, v) \\
y(u, v) \\
z(u, v)
\end{array}\right)=\left(\begin{array}{c}
\cos (2 \pi u) \sin (\pi v) \\
\sin (2 \pi u) \sin (\pi v) \\
\cos (\pi v)
\end{array}\right) \\
& =\sum_{k=0}^{M_{1}-1} \sum_{l=-1}^{M_{2}+1} c[k, l] \varphi_{M_{1}}\left(M_{1} u-k\right) \varphi\left(M_{2} v-l\right)
\end{aligned}
$$



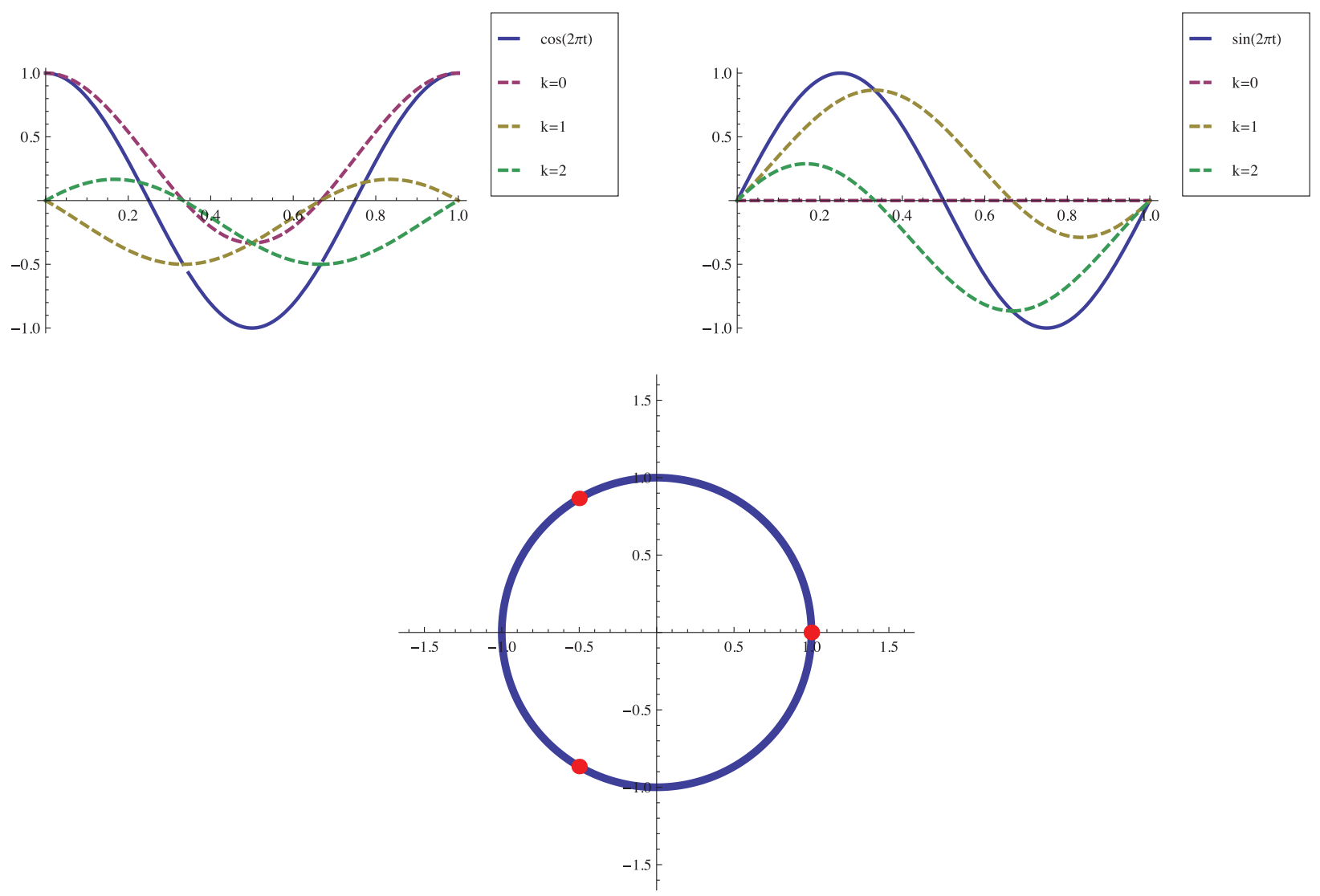

Fig. 4. The functions $\cos (2 \pi t)$ (top left) and $\sin (2 \pi t)$ (top right) are shown together respectively (blue curves) with the underlying shifted basis functions (dashed curves) that correspond to $M=3$ and the shift $k$. Note that, in the construction of the sine, the contribution of the basis function corresponding to the shift $k=0$ is zero because in (25) it is computed through $\sin \left[\frac{2 \pi k}{M}\right] \varphi_{M}(M t-k)=0 \cdot \varphi_{M}(M t)=0$. Bottom: Circle obtained with the parametric equation $\boldsymbol{r}(t)=(\cos (2 \pi t), \sin (2 \pi t))$. (For interpretation of the references to color in this figure, the reader is referred to the web version of this article.)

where $u, v \in[0,1]$ and the control points are given by

$$
c[k, l]=\left(\begin{array}{c}
\cos \left[\frac{2 \pi k}{M_{1}}\right] \sin \left[\frac{2 \pi l}{2 M_{2}}\right] \\
\sin \left[\frac{2 \pi k}{M_{1}}\right] \sin \left[\frac{2 \pi l}{2 M_{2}}\right] \\
\cos \left[\frac{2 \pi l}{2 M_{2}}\right]
\end{array}\right) .
$$

We choose $M_{1}=2 M_{2}$ because the term that depends on $u$ is 1-periodic while the term that depends on $v$ is 2-periodic. The fact that $l$ only needs to run from -1 to $M_{2}+1$ is due to the support of $\varphi$, which is equal to 4 . The result is shown in the top-left image of Fig. 5. For comparison, we are also displaying the solutions obtained by the non-interpolatory scheme described in [9]. While the displayed shapes are the same in both cases, the essential difference is that the control points of our proposed interpolators lie on the surface; a property that is useful if the shape needs to be modified interactively. Because each control point is associated to a limited number of compactly supported basis functions, moving its location results in a local modification of the surface.

\subsubsection{Torus}

The torus can be reproduced in a way similar to the sphere. Again, using the same basis function of our working example (18) and the standard parameterization of the torus, we obtain

$$
\begin{aligned}
\sigma(u, v) & =\left(\begin{array}{l}
x(u, v) \\
y(u, v) \\
z(u, v)
\end{array}\right)=\left(\begin{array}{c}
(R+r \cos (2 \pi v)) \cos (2 \pi u) \\
(R+r \cos (2 \pi v)) \sin (2 \pi u) \\
r \sin (2 \pi v)
\end{array}\right) \\
& =\sum_{k=0}^{M-1} \sum_{l=0}^{M-1} c[k, l] \varphi_{M}(M u-k) \varphi_{M}(M v-l),
\end{aligned}
$$



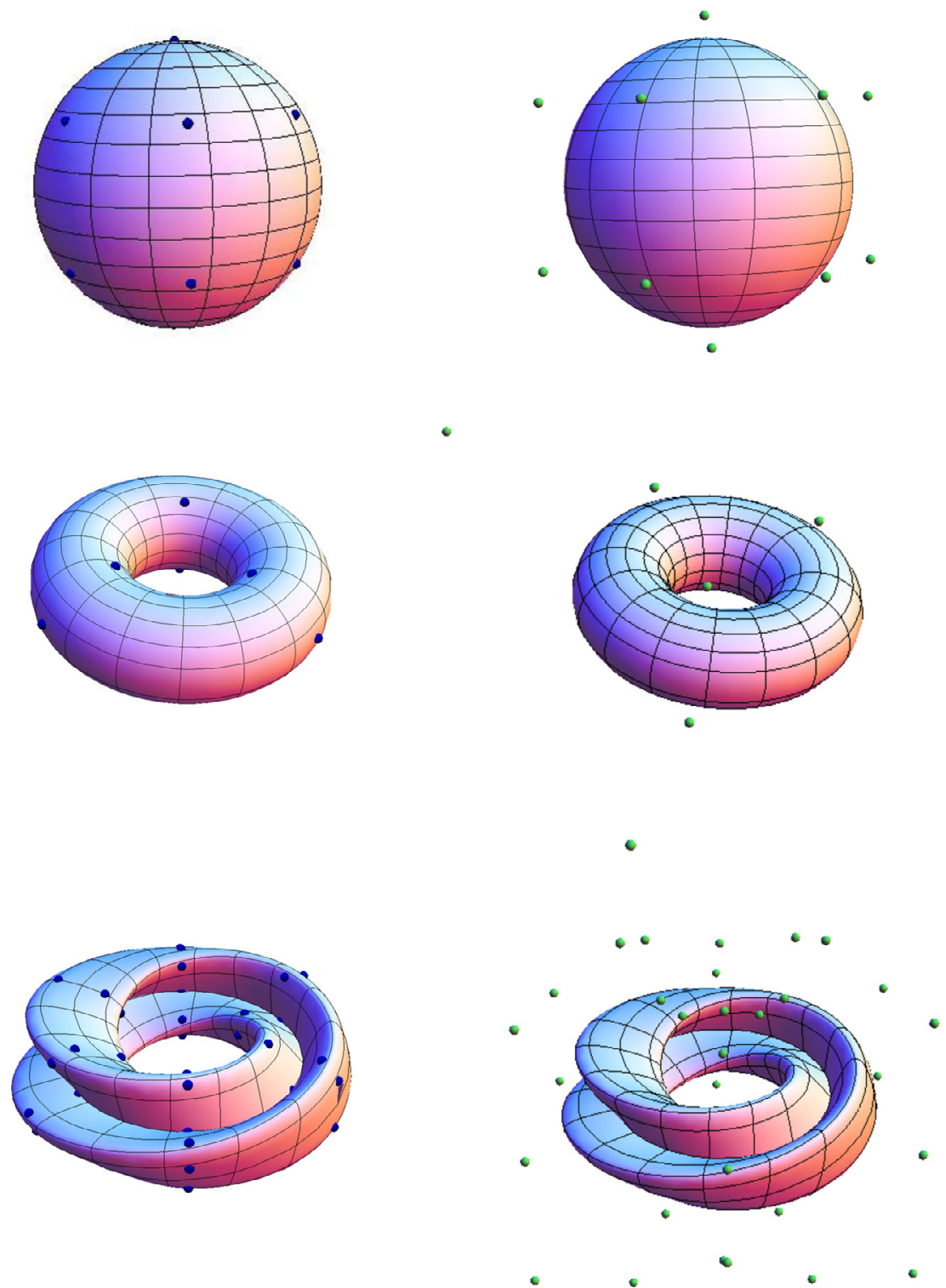

Fig. 5. Shape comparison. Interpolatory (left, blue) and non-interpolatory control points (right, green). The basis functions that were used to construct the noninterpolatory surfaces correspond to the ones presented in [13]. For the non-interpolatory surfaces it is difficult and non-intuitive to associate a given control point to the specific surface patch that is modified when moving the control point through user-interaction. (For interpretation of the references to color in this figure, the reader is referred to the web version of this article.)

where $u, v \in[0,1]$ and the control points are obtained by sampling as

$$
\left.\left(\begin{array}{c}
(R+r \cos (2 \pi v)) \cos (2 \pi u) \\
(R+r \cos (2 \pi v)) \sin (2 \pi u) \\
r \sin (2 \pi v)
\end{array}\right)\right|_{u=\frac{k}{M}, v=\frac{l}{M}}
$$

The radii $R$ and $r$ of the torus can be chosen in an arbitrary way, without affecting the shape but only the size of the surface. The resulting surface is shown in the middle-left image in Fig. 5. 


\subsection{3. "Figure 8" immersion}

The so-called "figure 8" immersion has a slightly more complicated parameterization than the two previous examples. It is given by

$$
\begin{aligned}
\sigma(u, v)= & \left(\begin{array}{l}
x(u, v) \\
y(u, v) \\
z(u, v)
\end{array}\right)=\left(\begin{array}{c}
(r+\cos (\pi u) \sin (2 \pi v)-\sin (\pi u) \sin (4 \pi v)) \cos (2 \pi u) \\
(r+\cos (\pi u) \sin (2 \pi v)-\sin (\pi u) \sin (4 \pi v)) \sin (2 \pi u) \\
\sin (\pi u) \sin (2 \pi v)+\cos (\pi u) \sin (4 \pi v)
\end{array}\right) \\
= & \left(\begin{array}{c}
r \cos (2 \pi u)+\frac{1}{2} \sin (2 \pi v)(\cos (\pi u)+\cos (3 \pi u))-\frac{1}{2} \sin (4 \pi v)(\sin (3 \pi u)-\sin (\pi u)) \\
r \sin (2 \pi u)+\frac{1}{2} \sin (2 \pi v)(\sin (\pi u)+\sin (3 \pi u))-\frac{1}{2} \sin (4 \pi v)(\cos (\pi u)-\cos (3 \pi u)) \\
\sin (\pi u) \sin (\pi v)+\cos (\pi u) \sin (4 \pi v)
\end{array}\right)
\end{aligned}
$$

where $u, v \in[0,1]$ and $r>2$ is a constant. Hence, we notice that the frequencies associated with the parameter $u$ are $\pi, 2 \pi$, and $3 \pi$, whereas the frequencies associated with $v$ are $2 \pi$ and $4 \pi$. Therefore, we construct two interpolators, $\varphi_{1}$ with $\boldsymbol{\alpha}_{\varphi_{1}}=$ $\left(\frac{\mathrm{j} \pi}{M},-\frac{\mathrm{j} \pi}{M}, \frac{\mathrm{j} 2 \pi}{M},-\frac{\mathrm{j} 2 \pi}{M}, \frac{\mathrm{j} 3 \pi}{M},-\frac{\mathrm{j} 3 \pi}{M}\right)$ and $\varphi_{2}$ with $\boldsymbol{\alpha}_{\varphi_{2}}=\left(\frac{\mathrm{j} 2 \pi}{M},-\frac{\mathrm{j} 2 \pi}{M}, \frac{\mathrm{j} 4 \pi}{M},-\frac{\mathrm{j} 4 \pi}{M}\right)$.

The expression for the tensor-product spline surface is then given by

$$
\boldsymbol{\sigma}(u, v)=\sum_{k=0}^{M_{1}-1} \sum_{l=0}^{M_{2}-1} \boldsymbol{c}[k, l] \varphi_{1, M_{1}}\left(M_{1} u-k\right) \varphi_{2, M_{2}}\left(M_{2} v-l\right),
$$

where $\varphi_{1}$ and $\varphi_{2}$ have been periodized and $\boldsymbol{c}[k, l]=\left.\sigma(u, v)\right|_{u=k, \nu=l}$.

The resulting surface is shown at the bottom left in Fig. 5.

\section{Conclusion}

We have characterized a new family of compactly supported interpolators that are based on exponential B-splines. We have shown that they reproduce exponential polynomials while being interpolating. We have illustrated how different members of the family, such as polynomial, trigonometric, or hyperbolic interpolators of different orders can be constructed according to desirable reproduction properties. We have also shown how the proposed interpolators can be used to represent parametric curves and surfaces. The interpolation property ensures that the control points lie on the curve or surface itself. This property is particularly useful for shape representation or manipulation in user-interactive applications. The proposed family of interpolating functions can be seen as a generalization of the polynomial Keys interpolator to higher orders as well as its extension with respect to the reproduction of exponential polynomials.

\section{References}

[1] E. Cohen, T. Lyche, R. Riesenfeld, Discrete B-splines and subdivision techniques in computer-aided geometric design and computer graphics, Comput Graph. Image Process. 14 (2) (1980) 87-111.

[2] L. Romani, M.A. Sabin, The conversion matrix between uniform B-spline and Bezier representations, Comput. Aided Geom. Des. 21 (6) (2004) 549-560.

[3] M. Audette, A. Chernikov, N. Chrisochoides, A review of mesh generation for medical simulators, Handb. Real-World Appl. Model. Simul. 2 (2012) 261.

[4] A. Garg, A. Sageman-Furnas, B. Deng, Y. Yue, E. Grinspun, M. Pauly, M. Wardetzky, Wire mesh design, ACM Trans. Graph. 33 (4) (2014) 66:1-66:12.

[5] B. Deng, S. Bouaziz, M. Deuss, A. Kaspar, Y. Schwartzburg, M. Pauly, Interactive design exploration for constrained meshes, Compu. Aid. Des. 61 (2015) $13-23$.

[6] T. DeRose, M. Kass, T. Truong, Subdivision surfaces in character animation, in: Proceedings of the 25th Annual Conference on Computer Graphics and Interactive Techniques, SIGGRAPH'98, ACM, New York, NY, USA, 1998, pp. 85-94.

[7] R. Delgado-Gonzalo, V. Uhlmann, D. Schmitter, M. Unser, Snakes on a plane: a perfect snap for bioimage analysis, IEEE Signal Process. Mag. 32 (1) (2015) 41-48.

[8] D. Schmitter, R. Delgado-Gonzalo, G. Krueger, M. Unser, Atlas-free brain segmentation in 3D proton-density-like MRI images, in: Proceedings of the Eleventh IEEE International Symposium on Biomedical Imaging: From Nano to Macro (ISBl'14), Beijing, People's Republic of China, 2014, pp. 629-632.

[9] R. Delgado-Gonzalo, N. Chenouard, M. Unser, Spline-based deforming ellipsoids for interactive 3D bioimage segmentation, IEEE Trans. Image Process. 22 (10) (2013) 3926-3940.

[10] L. Piegl, W. Tiller, The NURBS Book, 2nd ed., Springer, Berlin, Heidelberg, 2010.

[11] C.V. Beccari, G. Casciola, L. Romani, Construction and characterization of non-uniform local interpolating polynomial splines, J. Comput. Appl. Math. 240 (2013) 5-19.

[12] M. Antonelli, C. Beccari, G. Casciola, A general framework for the construction of piecewise-polynomial local interpolants of minimum degree, Adv. Comput. Math. 40 (4) (2014) 945-976.

[13] R. Delgado-Gonzalo, P. Thévenaz, M. Unser, Exponential splines and minimal-support bases for curve representation, Comput. Aid. Geom. Des. 29 (2) (2012) 109-128.

[14] N. Dyn, E. Farkhi, Spline subdivision schemes for compact sets. A survey, Serdica Math. J. 28 (2002) 349-360.

[15] C. Conti, L. Romani, Algebraic conditions on non-stationary subdivision symbols for exponential polynomial reproduction, J. Comput. Appl. Math. 236 (4) (2011) 543-556

[16] M. Charina, C. Conti, L. Romani, Reproduction of exponential polynomials by multivariate non-stationary subdivision schemes with a general dilation matrix, Numerische Mathematik 127 (2) (2014) 223-254.

[17] P. Novara, L. Romani, Building blocks for designing arbitrarily smooth subdivision schemes with conic precision, J. Comput. Appl. Math. 279 (0) (2015) 67-79.

[18] C. Conti, L. Romani, M. Unser, Ellipse-preserving Hermite interpolation and subdivision, J. Math. Anal. Appl. 426 (1) (2015) $211-227$.

[19] N. Dyn, L. David, A. Luzzatto, Exponentials reproducing subdivision schemes, Found. Comput. Math. 3 (2) (2003) $187-206$.

[20] R. Keys, Cubic convolution interpolation for digital image processing, IEEE Trans. Acoustics Speech Signal Process. 29 (6) (1981) 1153-1160.

[21] E. Catmull, R. Rom, A class of local interpolating splines, Comput. Aided Geom. Des., Academic Press, 1974, pp. 317-326.

[22] E. Meijering, M. Unser, A note on cubic convolution interpolation, IEEE Trans. Image Process. 12 (4) (2003) 477-479. 
[23] M. Unser, T. Blu, Cardinal exponential splines: part I-Theory and filtering algorithms, IEEE Trans. Signal Process. 53 (4) (2005) $1425-1438$.

[24] M. Unser, Cardinal exponential splines: part II-Think analog, act digital, IEEE Trans. Signal Process. 53 (4) (2005) 1439-1449.

[25] G. Strang, G. Fix, A Fourier analysis of the finite element variational method, Constr. Asp. Funct. Anal. (1973) 793-840.

[26] T. Blu, P. Thévenaz, M. Unser, Complete parameterization of piecewise-polynomial interpolation kernels, IEEE Trans. Image Process. 12 (11) (2003) 12971309.

[27] R. Delgado-Gonzalo, P. Thévenaz, C. Seelamantula, M. Unser, Snakes with an ellipse-reproducing property, IEEE Trans. Image Process. 21 (3) (2012) 12581271. 